ARTICLE

\title{
Photoresponsive supramolecular coordination polyelectrolyte as smart anticounterfeiting inks
}

\author{
Zhiqiang Li ${ }^{1 凶}$, Xiao Liư ${ }^{1}$, Guannan Wang ${ }^{1}$, Bin $\mathrm{Li}^{1}$, Hongzhong Chen² , Huanrong $\mathrm{Li}^{1 凶} \&$ Yanli Zhao (1) ${ }^{2 凶}$
}

While photoluminescence printing is a widely applied anticounterfeiting technique, there are still challenges in developing new generation anticounterfeiting materials with high security. Here we report the construction of a photoresponsive supramolecular coordination polyelectrolyte (SCP) through hierarchical self-assembly of lanthanide ion, bis-ligand and diarylethene unit, driven by metal-ligand coordination and ionic interaction. Owing to the conformation-dependent photochromic fluorescence resonance energy transfer between the lanthanide donor and diarylethene acceptor, the ring-closure/ring-opening isomerization of the diarylethene unit leads to a photoreversible luminescence on/off switch in the SCP. The SCP is then utilized as security ink to print various patterns, through which photoreversible multiple information patterns with visible/invisible transformations are realized by simply alternating the irradiation with UV and visible light. This work demonstrates the possibility of developing a new class of smart anticounterfeiting materials, which could be operated in a noninvasive manner with a higher level of security.

\footnotetext{
${ }^{1}$ Tianjin Key Laboratory of Chemical Process Safety, School of Chemical Engineering and Technology, Hebei University of Technology, Tianjin, P. R. China. ${ }^{2}$ Division of Chemistry and Biological Chemistry, School of Physical and Mathematical Sciences, Nanyang Technological University, Singapore, Singapore.

凶email: zhiqiangli@hebut.edu.cn; lihuanrong@hebutedu.cn; zhaoyanli@ntu.edu.sg
} 
C ounterfeit goods such as currency, microelectronics, software, movie films, pharmaceutics, and clothing in the market not only cause economic loss to customer and copyright owners, but also bring potential risks to the health and lives of consumers ${ }^{1-3}$. Governments and copyright holders are forced to increase their investments in developing anticounterfeiting technologies. The global market size of anticounterfeiting technologies was 51.8 billion USD in 2017, and the global anticounterfeiting packaging market is expected to grow to 208.4 billion USD in $2023^{4}$. Amongst the anticounterfeiting techniques and signal outputs, photoluminescence printing is the most widely applied one, because it offers advantages such as easy handling, high-throughput, facile design, and tunable optical properties in multiple dimensions ${ }^{5-7}$. For instance, a series of optical materials, including but not limited to upconversion nanoparticles ${ }^{8,9}$, organic dyes ${ }^{10-12}$, quantum dots ${ }^{13}$, metalorganic frameworks ${ }^{14,15}$, and perovskites ${ }^{16,17}$, are promising candidates as anticounterfeiting taggants. Lanthanide complexes are also widely applied in anticounterfeiting due to their inherent optical properties, including distinguishable spectroscopic fingerprint, large Stokes shift, and long excited lifetime ${ }^{18-22}$. For example, $\mathrm{Eu}^{2+} / \mathrm{Eu}^{3+}$ are used in Euro banknotes as a luminescence anticounterfeiting label ${ }^{23}$.

However, there are still several challenges in developing new generations of anticounterfeiting materials with more covert and reliable features capable of providing higher security level. (1) Quit a large number of luminescent inks are suspended/dissolved in organic solvents, or contain toxic ions, thus limiting their applications in authenticating food and medicine ${ }^{4,24}$. (2) Authentic information recorded in materials with static optical outputs is often visible under ambient condition or the excitation of UV light ${ }^{25-27}$. Thus, stimulusresponsive materials that can respond to external stimuli and alter their optical outputs would be ideal to bring additional security features, making them more difficult to forge ${ }^{2,28-31}$. On the other hand, invasive stimulus approaches (e.g., thermal, chemical, and mechanical means) may not only contaminate or destroy the goods, but also be inconvenient to operate ${ }^{32-37}$. For example, it is unrealistic for untrained consumer to add acid, alkali or other chemicals to the labels by themselves. Heating approach may cause damage to goods. 3) In terms of printing technologies, inkjet printing is the most common printing form today ${ }^{38}$. In many cases, however, the widespread use of inkjet printing fluorescent nanoparticles/nanocrystals requires either complicated assembly and coating procedures to achieve sufficient loading of nanoparticles and long-term stability of the inks, or the modification of preexisting commercial inkjet printers to cope with high viscosity inks or inks containing oversized nanoparticles (such as aerosol jet printers) ${ }^{39-42}$.

To address above-discussed issues, herein, we developed a photoresponsive supramolecular coordination polyelectrolyte (SCP) via the electrostatic interactions of an anionic lanthanide coordination polymer with a cationic photochrome (Fig. 1). Reversible on/off switching of the luminescence signal was realized by remotely alternating UV and visible light irradiation, allowing the fabrication of anticounterfeiting tags for multipletime verifications. The anionic lanthanide coordination polymer was prepared by the coordination between $\mathrm{Eu}^{3+}$ and alkyl bridged bis-2,6-pyridinedicarboxylic acid ligand, followed by mixing with a cationic diarylethene derivative to form SCP in pure water. The diarylethene unit with the features of high photoisomerization yield, excellent fatigue resistance, and thermal irreversibility was chosen as a photoswitch ${ }^{43-47}$, since the photochromic fluorescence resonance energy transfer (FRET) between $\mathrm{Eu}^{3+}$ and diarylethene unit is typically governed by the conformation of diarylethene $e^{48-50}$. Thus, the as-prepared SCP exhibits characteristic emission of $\mathrm{Eu}^{3+}$, because the emission spectrum of $\mathrm{Eu}^{3+}$ does not overlap with the absorption spectrum of open-form diarylethene. The irradiation of SCP with UV light leads to the isomerization of open-form diarylethene to its closeform conformation, whose absorption band perfectly overlaps with the emission band of $\mathrm{Eu}^{3+}$. As a result, the luminescence is quenched due to the activation of the photochromic FRET between $\mathrm{Eu}^{3+}$ and photocyclized diarylethene. After subsequent visible light irradiation, the close-form diarylethene isomerizes back to its open-form, and the luminescence intensity is totally recovered. According to this unique property, SCP was filled into a commercially available desktop inkjet printer cartridge to print various high-resolution anticounterfeiting marks. Reversible authentic information with visible/invisible transformation was thus achieved by simple light stimuli, making it suitable for highsecurity anticounterfeiting applications. Hence, the ring-close and ring-open photoisomerization of the diarylethene moiety regulates the FRET process, leading to reversible luminescence on/off switch in SCP capable of multiple information authentication. In this system, both the lanthanide coordination polymer and the diarylethene derivative are water soluble, and thus, water is the only solvent used in preparing the security ink, enabling its usage in a green condition and its good compatibility with commercial printers. In addition, light irradiation offers clear triggers and spatiotemporal control over the anticounterfeiting patterns in a noninvasive manner.

\section{Results}

Synthesis and characterization. Bis-2,6-pyridinedicarboxylic acid ligand (L) was synthesized by a two-step procedure and comprehensively characterized (Supplementary Methods and Supplementary Figs. 1-7). Luminescence titration revealed that the coordination stoichiometry between 2,6-pyridinedicarboxylic acid (DPA) and $\mathrm{Eu}^{3+}$ is 3:1 (Supplementary Fig. 8), which is inconsistent with the previous report ${ }^{51,52}$. Trimeric lanthanide coordination polymer $\left(\mathrm{Eu}^{3+}-\mathbf{L}\right)$ was prepared by mixing compound $\mathrm{L}$ and $\mathrm{EuCl}_{3}$ in water with a molar ratio of $1.5: 1$ and characterized by FTIR spectra (Supplementary Fig. 9) and ${ }^{1} \mathrm{H}$ NMR spectra. Compared to individual $\mathbf{L}$, the absorption band at $1724 \mathrm{~cm}^{-1}$ assigned to the $\mathrm{C}=\mathrm{O}$ stretching vibration of DPA underwent a red shift to $1625 \mathrm{~cm}^{-1}$ in the FTIR spectrum of $\mathrm{Eu}^{3+}-\mathbf{L}$, implying the successful coordination of DPA with $\mathrm{Eu}^{3+}$ ion $^{53}$. In the ${ }^{1} \mathrm{H}$ NMR spectra (Fig. $2 \mathrm{a}, \mathrm{b}$ ), the proton signals assigned to ligand $\mathbf{L}$ became highly broadening after the coordination with $\mathrm{Eu}^{3+}$, further confirming the formation of the coordination polymer. The high coordination number of $\mathrm{Eu}^{3+}-\mathbf{L}$ not only benefits to sufficient sensitization of the $\mathrm{Eu}^{3+}$ ion based on the antenna effect, but also prevents luminescent quenching caused by the infiltration of water molecule, thus endowing the lanthanide coordination polymer with characteristic emission color and brightness in both aqueous solution and the solid state under UV light excitation (Supplementary Figs. 10, 11) ${ }^{54}$. The luminescence quantum yield of $\mathrm{Eu}^{3+}-\mathbf{L}$ aqueous solution was measured to be $23.31 \%$. The imidazolium salt modified openform diarylethene (OF-1) was synthesized through a robust twostep procedure in a yield of $72 \%$, along with full characterizations (Supplementary Figs. 12-21). UV-Vis (Supplementary Figs. 2226) and ${ }^{1} \mathrm{H}$ NMR spectra (Supplementary Figs. 27, 28) revealed that compound $\mathbf{1}$ had excellent reversible ring-open/ring-close photoisomerization behavior (Supplementary Notes 1 and 2).

The lanthanide coordination polymer carries three negative net charges $\left(6 \mathrm{COO}^{-}+\mathrm{Eu}^{3+}\right)$ per coordination center, allowing it to further assemble with positively charged OF-1 based on electrostatic interaction ${ }^{55-59}$. The SCP $\left(\mathrm{Eu}^{3+}-\mathrm{L}-\mathrm{OF}-1\right)$ was then prepared by mixing $\mathrm{Eu}^{3+}-\mathbf{L}$ and $\mathrm{OF}-\mathbf{1}$ at charge stoichiometry $\left(\mathrm{Eu}^{3+}: \mathrm{OF}-\mathbf{1}=1: 1.5\right)$. Zeta potential experiments were carried out to verify the existence of electrostatic interaction between $\mathrm{Eu}^{3+}-\mathbf{L}$ 


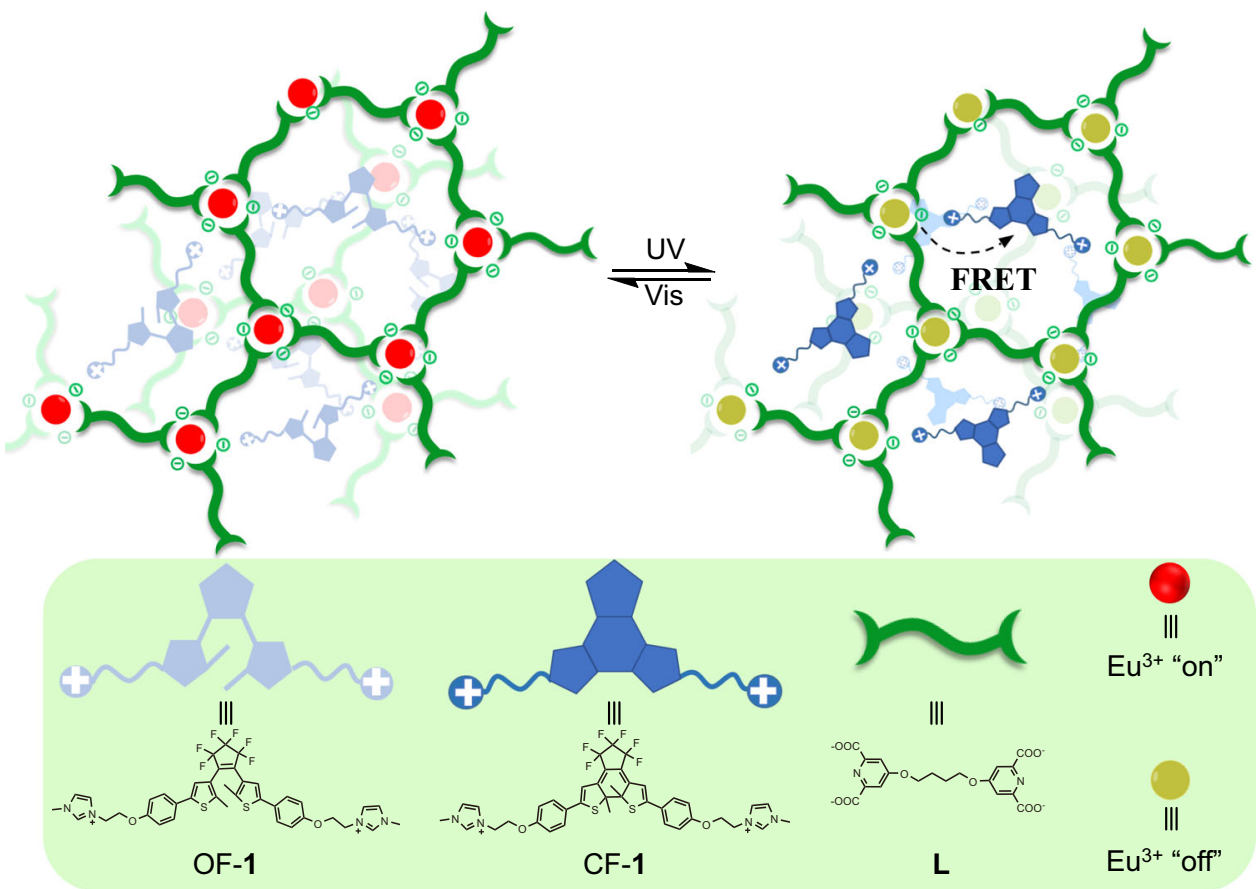

Fig. 1 Schematic illustration. The construction of the photochromic supramolecular coordination polyelectrolyte, and the chemical structures of corresponding components.

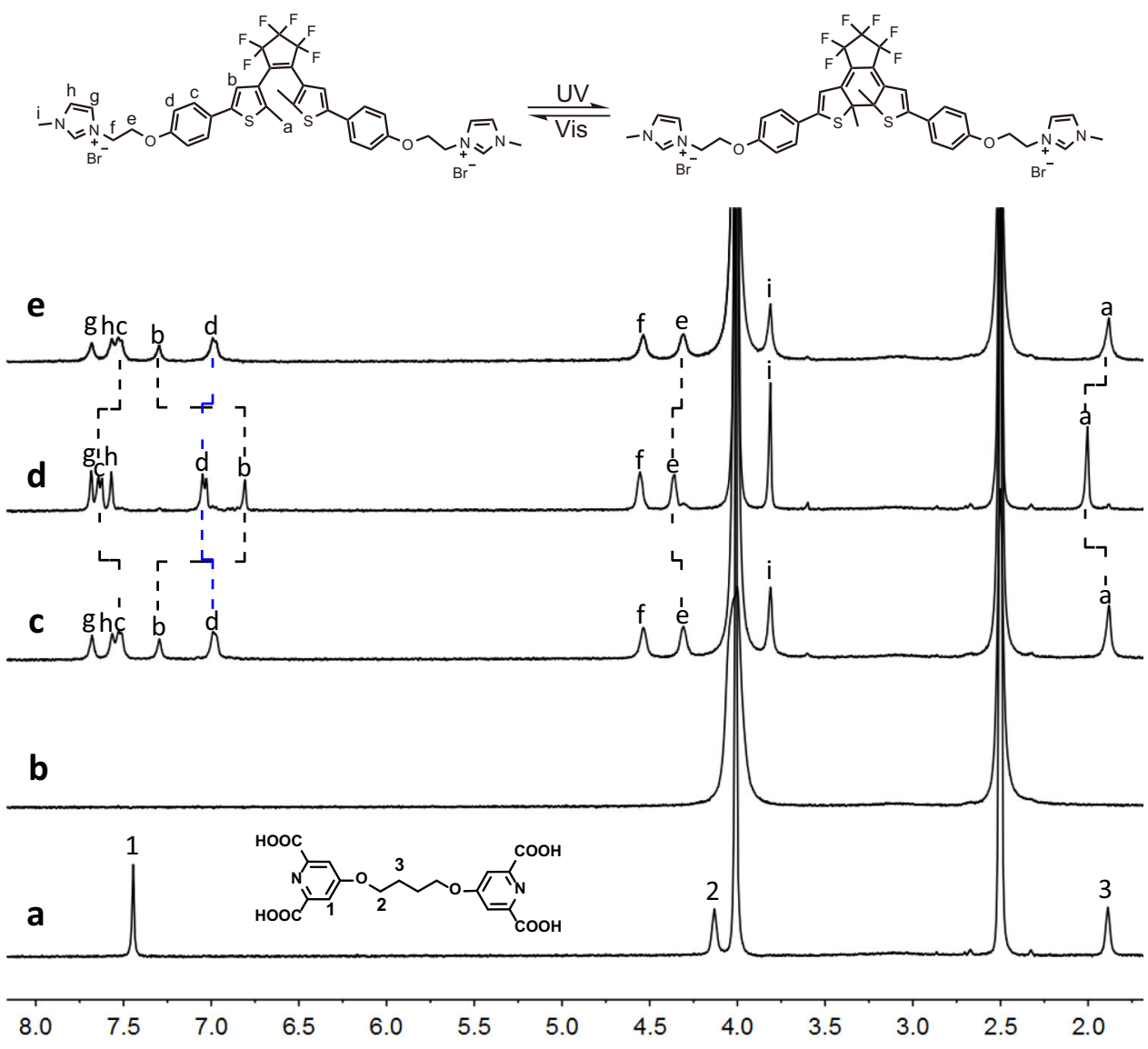

Chemical shift (ppm)

Fig. $2{ }^{1} \mathbf{H}$ NMR spectral studies. Partial ${ }^{1} \mathrm{H}$ NMR spectra (DMSO:D ${ }_{2} \mathrm{O}=4: 1,400 \mathrm{MHz}, 25^{\circ} \mathrm{C}$ ) of a compound $\mathbf{L}, \mathbf{b}$ Eu ${ }^{3+}-\mathbf{L}, \mathbf{c}-\mathbf{e}$ Eu ${ }^{3+}-\mathbf{L}-\mathrm{OF}-\mathbf{1}: \mathbf{c}$ before and d after the irradiation by UV light $(300 \mathrm{~nm}, 60 \mathrm{~min})$, and e subsequent irradiation with visible light $(>450 \mathrm{~nm}, 60 \mathrm{~min}) .\left[\mathrm{Eu}^{3+}\right]=1.4 \times 10^{-4} \mathrm{M},[\mathbf{L}]=[\mathrm{OF}-$ 1] $=2.1 \times 10^{-4} \mathrm{M}$. 

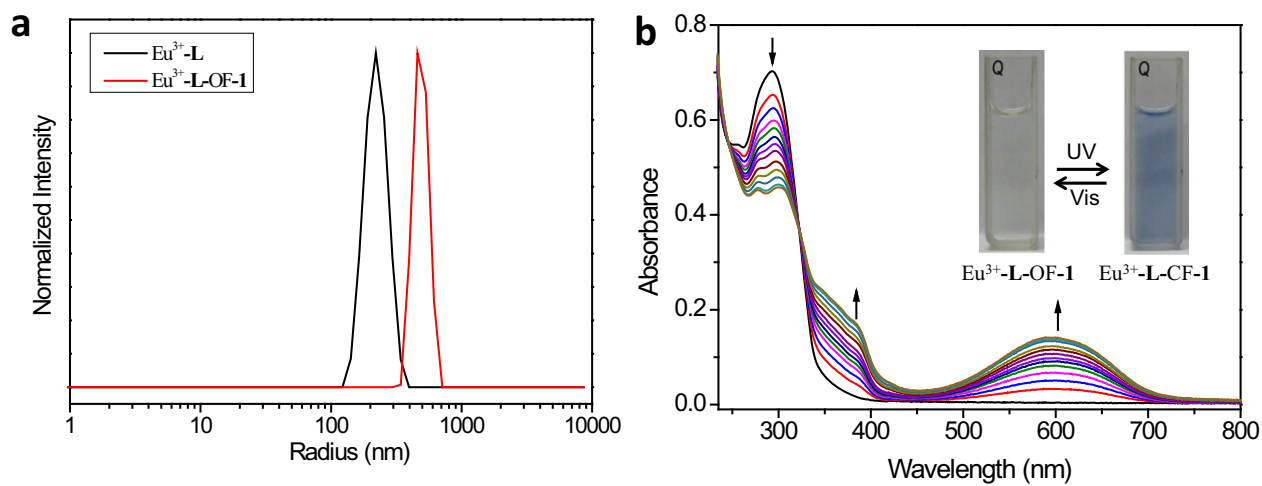

Fig. 3 DLS size distribution and UV-Vis spectral studies. a DLS size distribution of $\mathrm{Eu}^{3+}-\mathbf{L}$ (black curve) and Eu ${ }^{3+}-\mathbf{L}-\mathrm{OF}-\mathbf{1}\left(\mathrm{red}_{\mathrm{curve}}\right)\left(\left[\mathrm{Eu} \mathrm{u}^{3+}\right]=1.4 \times 10^{-4}\right.$ $\left.\mathrm{M},[\mathbf{L}]=[\mathrm{OF}-\mathbf{1}]=2.1 \times 10^{-4} \mathrm{M}\right) . \mathbf{b} \mathrm{UV}-\mathrm{Vis}$ spectral changes and corresponding photographic images of Eu ${ }^{3+}-\mathbf{L}-\mathrm{OF}-\mathbf{1}$ and Eu ${ }^{3+}-\mathbf{L}-\mathrm{CF}-\mathbf{1}$ with alternating $300 \mathrm{~nm}$ UV and $>450 \mathrm{~nm}$ visible light irradiation in water for up to $60 \mathrm{~s}$ each time $\left(\left[\mathrm{Eu}^{3+}\right]=1.4 \times 10^{-5} \mathrm{M},[\mathbf{L}]=[\mathrm{OF}-\mathbf{1}]=2.1 \times 10^{-5} \mathrm{M}\right)$.

and OF-1 (Supplementary Fig. 29). Individual $\mathrm{Eu}^{3+}-\mathbf{L}$ displayed a negative potential of $-19.53 \mathrm{mV}$, while the $\zeta$-potential value of individual OF-1 was measured to be $20.15 \mathrm{mV}$. The $\mathrm{Eu}^{3+}$-L-OF-1 solution was almost electrically neutral $(1.54 \mathrm{mV})$. These results confirmed the presence of electrostatic interaction between $\mathrm{Eu}^{3+}$. $\mathbf{L}$ and OF-1, enabling suitable distance between the energy donor and acceptor. Dynamic light scattering (DLS) measurements confirmed the formation of supramolecular assembly between $\mathrm{Eu}^{3+}-\mathbf{L}$ and OF-1. The DLS experiment of $\mathrm{Eu}^{3+}-\mathbf{L}$ (Fig. 3a) shows a hydrodynamic radius of $220 \mathrm{~nm}$, indirectly proving the formation of large-scaled coordination polymer in solution. The hydrodynamic radius of $\mathrm{Eu}^{3+}-\mathrm{L}-\mathrm{OF}-1$ increases to around $500 \mathrm{~nm}$, much larger than that of $\mathrm{Eu}^{3+} \mathbf{L}$, revealing that $\mathrm{Eu}^{3+}$. $\mathbf{L}$ assembles with OF-1 to form the supramolecular polymer ${ }^{60,61}$. Meanwhile, uniform spheres with an average diameter of $300 \mathrm{~nm}$ were observed by transmission electron microscopy (Supplementary Fig. 30), providing intuitive evidence for the formation of the self-assembly between $\mathrm{Eu}^{3+}-\mathbf{L}$ and OF-1.

Photoresponsive property. We then investigated the photoresponsive property of $\mathrm{Eu}^{3+}-\mathrm{L}-\mathrm{OF}-\mathbf{1}$ resulting from the isomerization of the diarylethene moiety. The UV-Vis spectra of $\mathrm{Eu}^{3+}$-L-OF-1 (Fig. 3b) showed an absorption band at $294 \mathrm{~nm}$ corresponding to OF-1 unit, and no absorption over $400 \mathrm{~nm}$ was observed. Upon the irradiation with UV light $(300 \mathrm{~nm})$, the absorption at $294 \mathrm{~nm}$ gradually decreased, two new absorption bands centered at $380 \mathrm{~nm}$ and $596 \mathrm{~nm}$ appeared. Meanwhile, the colorless aqueous solution changed to dark blue (insert of Fig. 3b). These phenomena jointly demonstrated the OF-1 transformed to its close form (CF-1) after the irradiation. All these changes levelled off in $60 \mathrm{~s}$ (Supplementary Fig. 31). Moreover, a well-defined isosbestic point was observed at $323 \mathrm{~nm}$, indicating that ring-open isomer cleanly transformed into the photocyclized form in $\mathrm{SCP}^{62,63}$. We further measured the photocyclization yield at the photostationary state by ${ }^{1} \mathrm{H}$ NMR spectra (Fig. 2c,d). Since the proton signals of OF-1 showed serious broadening in aqueous media (Supplementary Fig. 32), the ${ }^{1} \mathrm{H}$ NMR spectral study was carried out in mixed deuterated solvent (DMSO- $\mathrm{d}_{6}: \mathrm{D}_{2} \mathrm{O}=4: 1$ ). After irradiated by UV light $(300 \mathrm{~nm}, 60 \mathrm{~min})$, the thiophene protons $\left(\mathrm{H}_{\mathrm{b}}\right)$ underwent an obvious upfield shift from 7.30 to $6.81 \mathrm{ppm}$, mainly due to the electronic shielding effect in the large conjugated closed ring isomers ${ }^{64}$. The methyl protons $\left(\mathrm{H}_{\mathrm{a}}\right)$ of the diarylethene unit underwent an apparent downfield shift from 1.88 to $2.00 \mathrm{ppm}$. Meanwhile, the aromatic protons $\mathrm{H}_{\mathrm{c}}$ and $\mathrm{H}_{\mathrm{d}}$ showed downfield shifts from $7.52 \mathrm{ppm}$ to $7.63 \mathrm{ppm}$ and from $6.98 \mathrm{ppm}$ to
7.04 ppm, respectively. All these shifts were thorough, and no apparent residual peaks retained in the original chemical shifts after the UV light irradiation (Supplementary Fig. 33). The molar ratio of CF-1: OF-1 was determined to be 0.94:0.06 according to the integrating resonance of protons $\mathrm{H}_{\mathrm{a}}$, indicating nearly quantitative $(\sim 94 \%)$ conversion from $\mathrm{Eu}^{3+}-\mathbf{L}-\mathrm{OF}-1$ to $\mathrm{Eu}^{3+}-\mathbf{L}-$ CF-1 upon exposure to UV light ${ }^{65}$. Interestingly, a complete recovery in both UV-Vis (Supplementary Figs. 34, 35) and ${ }^{1} \mathrm{H}$ NMR spectra (Fig. 2e) was achieved upon subsequent irradiation of the resulting $\mathrm{Eu}^{3+}-\mathrm{L}-\mathrm{CF}-1$ solution with $>450 \mathrm{~nm}$ visible light, accompanied by color change back to colorless, revealing that this photoisomerization behavior was fully reversible.

The photoresponsive luminescent behavior of SCP was then investigated. In the as-prepared $\mathrm{Eu}^{3+}-\mathrm{L}-\mathrm{OF}-\mathbf{1}$, no FRET was observed, because there was no spectral overlapping between the UV-Vis absorption of OF-1 and the emission spectrum of $\mathrm{Eu}^{3+}-\mathbf{L}$. $\mathrm{Eu}^{3+}$-L-OF-1 exhibited the characteristic spectral line of lanthanide. The excitation spectrum of $\mathrm{Eu}^{3+}-\mathrm{L}-\mathrm{OF}-\mathbf{1}$ showed a broad band centered at $265 \mathrm{~nm}$, attributed to the absorption of the DPA moiety (Supplementary Fig. 36). The corresponding emission spectrum was composed of five sharp peaks at 580, 594, 615, 649, and $692 \mathrm{~nm}$, referred to the ${ }^{5} \mathrm{D}_{0}$ to ${ }^{7} \mathrm{~F}_{\mathrm{J}}(J=0-4)$ transitions of $\mathrm{Eu}^{3+}$ respectively, in which the ${ }^{5} \mathrm{D}_{0} \rightarrow{ }^{7} \mathrm{~F}_{2}$ transition at $615 \mathrm{~nm}$ is dominant and responsible for the bright red emitting color (Supplementary Fig. 36) ${ }^{66}$. On the other hand, the luminescence emission spectrum of lanthanide coordination polymer $\mathrm{Eu}^{3+}-\mathbf{L}$ completely overlapped with the absorption spectrum of CF-1 in the range of 500-700 $\mathrm{nm}$ (Fig. 4a), implying that efficient FRET process may occur from $\mathrm{Eu}^{3+}$ to $\mathrm{CF}-\mathbf{1}$ in $\mathrm{Eu}^{3+}-\mathbf{L}-\mathrm{CF}-\mathbf{1}$. As expected, the luminescence of $\mathrm{Eu}^{3+}$ (Fig. 4b) was quenched gradually upon irradiating SCP with UV light. The luminescence quenching followed a biexponential attenuation law, containing a fast process, followed by a slow process to the photostationary state in $60 \mathrm{~s}$ (inset of Fig. $4 \mathrm{~b})^{49}$. The luminescence intensity was quenched completely at the end, and the decay decreased from 1,289 to $12 \mu \mathrm{s}$ (Supplementary Figs. 37-41), with concomitant decrease of the luminescence quantum yield from $15.84 \%$ to $0.85 \%$. These phenomena confirmed the occurrence of the FRET process with an efficiency $(E)$ of $98 \%$, calculated according to the reported method ${ }^{67}$. The quenched luminescence of $\mathrm{Eu}^{3+}$-L-CF-1 could completely recover to its original level upon subsequent visible light irradiation, ascribing to the photocycloreversion reaction (Fig. 4c). In particular, the photocontrolled luminescence on/off switch of SCP presented outstanding reversibility, and no apparent deterioration in the luminescence intensity (less than 4\%) was observed after 20 consecutive cycles of alternating UV and visible light irradiations (Fig. 4d). Thus, SCP exhibited excellent 

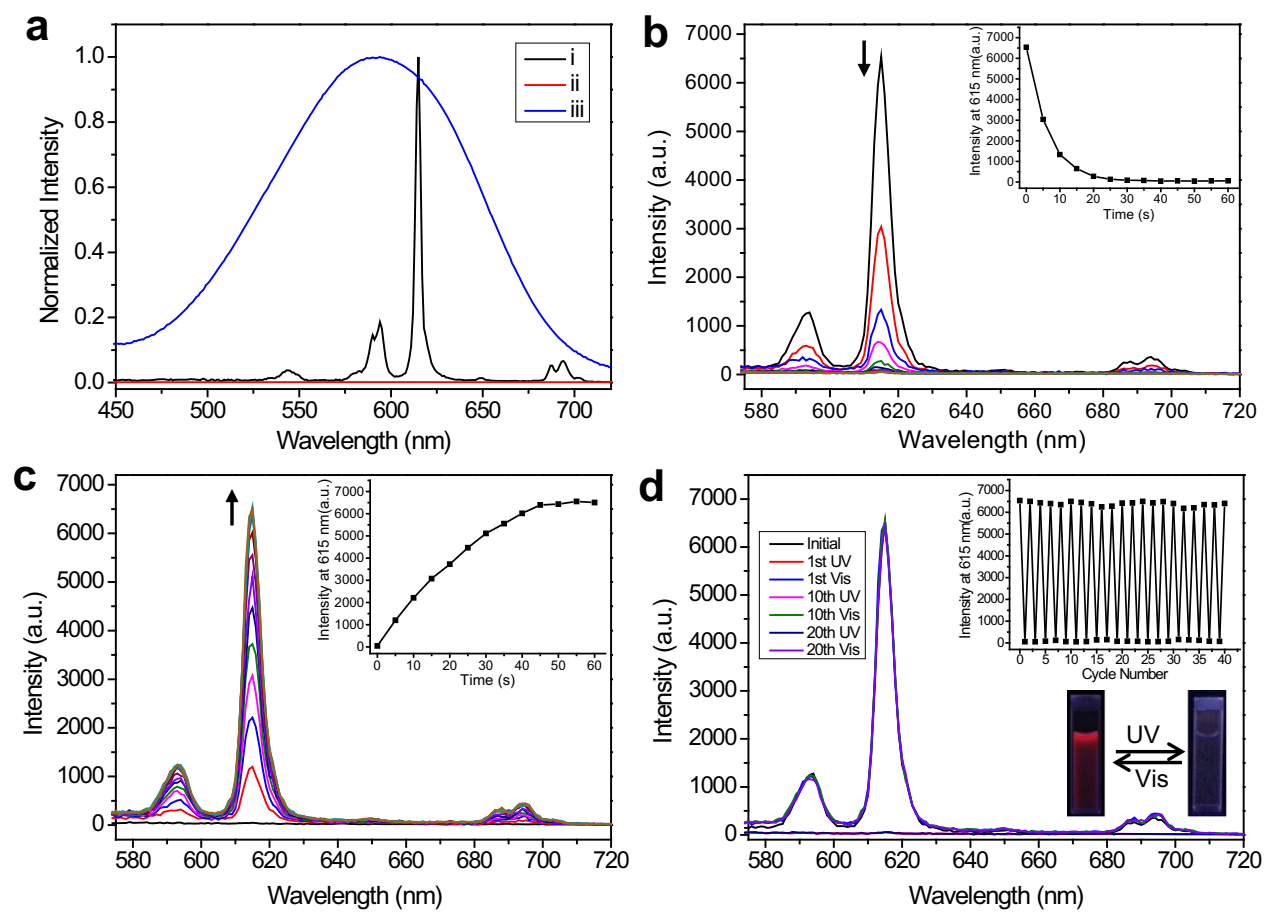

Fig. 4 Photophysical studies. a Partial emission spectrum (black curve) of Eu ${ }^{3+}-\mathbf{L}$, and absorption spectra of OF-1 (red curve) before and (blue curve) after irradiation with $300 \mathrm{~nm}$ UV light for $60 \mathrm{~s}$. b, c Luminescence emission spectral changes of Eu ${ }^{3+}$-L-OF-1 upon (b) UV light (300 nm) irradiation and c subsequent visible light (>450 nm) irradiation in water. Insets show corresponding emission intensity changes at $615 \mathrm{~nm}$. d Luminescence emission changes of Eu ${ }^{3+}$-L-OF-1 upon consecutive alternating exposure to UV and visible light. Insets show corresponding intensity changes at $615 \mathrm{~nm}$ (upper) and the photographs of the SCP solution under $254 \mathrm{~nm}$ UV lamp (lower). $\left[\mathrm{Eu}^{3+}\right]=1.4 \times 10^{-4} \mathrm{M}$, $[\mathbf{L}]=[\mathrm{OF}-\mathbf{1}]=2.1 \times 10^{-4} \mathrm{M}$.

fatigue resistance, which is of utmost importance for multiple anticounterfeiting applications.

It is worth noticing that the diarylethene derivative is bistable ${ }^{37}$, which means that the spontaneous photocycloreversion reaction is extremely slow under natural conditions. The half-life $\left(t_{1 / 2}\right)$ of Eu ${ }^{3+}$-L-CF- 1 at $25^{\circ} \mathrm{C}$ was estimated to be 376.7 min (Supplementary Figs. 42, 43, Supplementary Table 1, and Supplementary Note 3), ranking one of the longest $t_{1 / 2}$ values reported so far in diarylethene derivatives ${ }^{68,69}$, which confirmed that the self-switching is negligible. Only slight self-switching of $\mathrm{Eu}^{3+}$-L-CF-1 was observed upon continuous exposure to sunlight for $90 \mathrm{~min}$ (Supplementary Fig. 44). When the $\mathrm{Eu}^{3+}{ }_{-\mathrm{L}} \mathrm{C}-\mathrm{CF}-1$ solution was kept at an elevated temperature $\left(60^{\circ} \mathrm{C}\right)$ in the dark, no sign of thermal ring opening was observed from the UV-Vis spectra, supporting the good thermal stability of $\mathrm{Eu}^{3+}{ }_{-} \mathrm{L}-\mathrm{CF}-1$ (Supplementary Fig. 45).

Pattern printing. The developed SCP with important features of rapid response, prominent anti-fatigue capability and thermally irreversible luminescence on/off photoswitch encouraged us to further explore its performance in smart anticounterfeiting. We directly filled the $\mathrm{Eu}^{3+}$-L-OF-1 aqueous solution in a commercial inkjet printer (canon PIXMA ip1180) cartridge with the concentration low to $2.1 \times 10^{-4} \mathrm{M}$ (according to the concentration of OF-1), and printed various high-resolution quick response (QR) codes on commercial blue polyester terephthalate (PET) films (Fig. 5a, b). The obtained QR code was invisible under daylight due to the colorless nature of $\mathrm{Eu}^{3+}$-L-OF-1 aqueous solution (Fig. 5c and Supplementary Movie 1). However, bright red luminescent pattern was observed under $254 \mathrm{~nm}$ UV lamp, allowing to retrieve the encoded information quickly and accurately by scanning through a smartphone (Fig. $5 \mathrm{~d}$ and Supplementary Movie 2). It should be noted that the UV absorbance intensity of OF-1 at $254 \mathrm{~nm}$ is low, and thus the conversion from OF-1 to CF-1 under $254 \mathrm{~nm}$ UV lamp is very slow, providing enough time for recognizing the authentic information recorded in the QR code. The luminescence was quenched upon $300 \mathrm{~nm}$ UV light irradiation, making the QR code invisible under UV light. Although the pattern turned to blue under daylight, it can be completely masked by the blue background of the PET film. Through which, the absolutely and really invisible security pattern was achieved under both daylight (Fig. 5e and Supplementary Movie 3) and UV light (Fig. 5f and Supplementary Movie 4), which was highly sufficient for confidential information encryption.

As discussed above, CF-1 is the photostable state under daylight, especially in solid state. Consequently, the erased pattern remained unreadable even after placing under sunlight for one month (Supplementary Movies 5 and 6). The erased pattern could be completely recovered and recognized upon further irradiating with visible light $(>450 \mathrm{~nm})$. Moreover, even after 20 consecutive switching cycles, the quality of the remote light triggered information pattern with visible/invisible transformation process still remained unaffected (Supplementary Movies 7 and 8). Thus, the rapid response, noninvasive regulation, excellent fatigue resistance, and thermal irreversibility of SCPbased system made it a suitable anticounterfeiting ink for multiple authentic information encryption and decryption.

\section{Discussion}

In summary, we have developed a hierarchical self-assembly approach to realize a photoresponsive supramolecular coordination polyelectrolyte capable of reversible multiple information encryption and decryption. An anionic lanthanide coordination polymer, obtained from the coordination between lanthanide ion and a bis-ligand, further assembles with a cationic diarylethene 


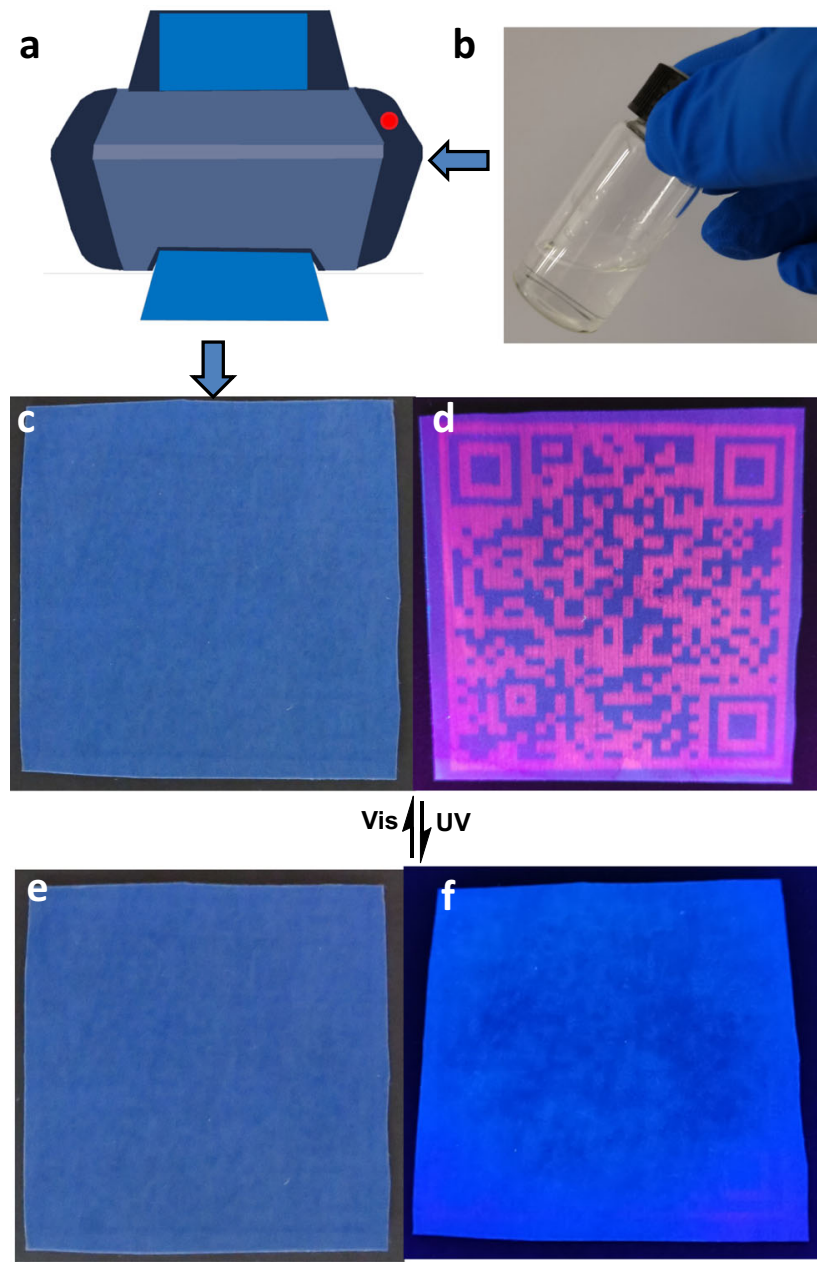

Fig. 5 Pattern printing using SCP as the ink. a, b Schematic illustration of the pattern printing process. Light triggered QR code with visible/invisible transformation behavior was achieved by using supramolecular coordination polyelectrolyte (SCP) as the smart ink. c-f Digital photos of SCP-based luminescent QR code on commercial blue PET film (size: $5 \times 5$ $\mathrm{cm}$ ) upon alternating UV (300 nm, $60 \mathrm{~s})$ and visible light (>450 nm, $120 \mathrm{~s})$ irradiation. c, d Photos under daylight. e, f Photos under $254 \mathrm{~nm}$ UV lamp. $\left[\mathrm{Eu}^{3+}\right]=1.4 \times 10^{-4} \mathrm{M},[\mathbf{L}]=[\mathrm{OF}-\mathbf{1}]=2.1 \times 10^{-4} \mathrm{M}$ in the SCP ink.

derivative based on ionic interaction to afford the SCP. Significantly, the ring-open/ring-close photoisomerization of the diarylethene moiety governs the FRET process between the lanthanide emitting center and the diarylethene component, leading to reversible luminescence on/off switch in the SCP. This SCP has been directly utilized as a security ink to realize reversible authentic information patterning with visible/invisible transformation by simply alternating the exposure to UV and visible light. The developed materials and its associated patterning technology with environmentally friendly preparation process, remote light control, rapid response, excellent fatigue resistance and thermal irreversibility have demonstrated a promising potential as a highsecurity anticounterfeiting ink in various fields, including authenticating food and medicine.

\section{Methods}

Synthetic routes for compounds $\mathbf{L}$ and OF-1 are shown in Supplementary Figs. 1, 12.

Synthesis of compound 5. A mixture of compound 6 (474 mg, $1.98 \mathrm{mmol}$ ), 1,4-dibromobutane $(60 \mu \mathrm{L}, 0.5 \mathrm{mmol})$ and $\mathrm{K}_{2} \mathrm{CO}_{3}(248 \mathrm{mg}, 1.8 \mathrm{mmol})$ was stirred in $\mathrm{N}, \mathrm{N}$-dimethylformamide $(10 \mathrm{~mL})$ under $\mathrm{N}_{2}$ at $80^{\circ} \mathrm{C}$ for $48 \mathrm{~h}$. Then, the reaction mixture was poured into water $(100 \mathrm{~mL})$. The resulting white precipitate was collected and washed three times with water. The precipitate was then dissolved in $\mathrm{CH}_{2} \mathrm{Cl}_{2}(100 \mathrm{~mL})$ and washed with a solution of $5 \%$ aqueous $\mathrm{NaOH}$ solution $(2 \times 50 \mathrm{~mL})$. The organic phase was concentrated and dried under vacuum to give compound 5 as a white solid in $80 \%$ yield. ${ }^{1} \mathrm{H}$ NMR $\left(400 \mathrm{MHz}, \mathrm{CDCl}_{3}, \mathrm{ppm}\right): \delta$ $7.79(\mathrm{~s}, 4 \mathrm{H}), 4.48(\mathrm{q}, J=7.1 \mathrm{~Hz}, 8 \mathrm{H}), 4.27(\mathrm{~m}, 4 \mathrm{H}), 2.08(\mathrm{~m}, 4 \mathrm{H}), 1.46(\mathrm{t}, J=7.1 \mathrm{~Hz}$ 12H). ${ }^{13} \mathrm{C} \mathrm{NMR}\left(100 \mathrm{MHz}, \mathrm{CDCl}_{3}, \mathrm{ppm}\right): \delta 166.7,164.7,150.2,114.1,68.2,62.4$, 25.3, 14.2. HRMS $[\mathrm{M}+\mathrm{H}]^{+}$calcd. for $\mathrm{C}_{26} \mathrm{H}_{33} \mathrm{~N}_{2} \mathrm{O}_{10}+533.2135$; found: 533.2126 ; Anal. Cald. for $\mathrm{C}_{26} \mathrm{H}_{32} \mathrm{~N}_{2} \mathrm{O}_{10}$ : C, 58.64; H, 6.06; N, 5.26; Found: C, 58.58; H, $6.10 ; \mathrm{N}, 5.22$.

Synthesis of compound $\mathbf{L}$. A mixture of compound 5 (168 mg, $0.4 \mathrm{mmol}), \mathrm{KOH}$ (224 mg, $4 \mathrm{mmol})$, methanol $(10 \mathrm{~mL})$ and water $(10 \mathrm{~mL})$ was stirred at $60{ }^{\circ} \mathrm{C}$ for $12 \mathrm{~h}$, and then acidified with $\mathrm{HCl}(3 \mathrm{M})$ to $\mathrm{pH} 4$. The precipitate was collected by centrifugation, washed with $\mathrm{H}_{2} \mathrm{O}$, and dried under vacuum to give compound $\mathbf{L}$ as a white solid in $60 \%$ yield. ${ }^{1} \mathrm{H}$ NMR $\left(400 \mathrm{MHz}, \mathrm{D}_{2} \mathrm{O}, \mathrm{ppm}\right): \delta 7.59(\mathrm{~s}, 4 \mathrm{H}), 4.34$ (m, 4H), $2.08(\mathrm{~m}, 4 \mathrm{H}) .{ }^{13} \mathrm{C}$ NMR $\left(100 \mathrm{~Hz}, \mathrm{H}_{2} \mathrm{O}, \mathrm{ppm}\right): \delta$ 172.7, 166.7, 154.9, 111.4, 68.3, 24.7. HRMS [M-H] ${ }^{-}$calcd. for $\mathrm{C}_{18} \mathrm{H}_{15} \mathrm{~N}_{2} \mathrm{O}_{10}{ }^{-}$419.0727; found: 419.0734; Anal. Cald. for $\mathrm{C}_{18} \mathrm{H}_{16} \mathrm{~N}_{2} \mathrm{O}_{10}$ : C, 51.44; H, 3.84; N, 6.66; Found: C, 51.29; H, 3.78; N, 6.60 .

Preparation of the coordination polymer $\mathbf{E u}^{\mathbf{3}+} \mathbf{- L}$. Compound $\mathbf{L}$ ( $42 \mathrm{mg}, 0.1$ $\mathrm{mmol}$ ) and potassium hydroxide $(22.4 \mathrm{mg}, 0.4 \mathrm{mmol})$ were dissolved in water $(10 \mathrm{~mL})$. Then, europium chloride hexahydrate $(24.5 \mathrm{mg}, 0.067 \mathrm{mmol})$ was added with stirring for $30 \mathrm{~min}$. The mixture was dried under vacuum to give $\mathrm{Eu}^{3+}-\mathrm{L}$ as a white solid.

Synthesis of compound 4. 4-Hydroxyphenylboronicacidpinacolester (220 mg, $1 \mathrm{mmol}), 1,2$-dibromoethane $(940 \mathrm{mg}, 5 \mathrm{mmol})$, and $\mathrm{K}_{2} \mathrm{CO}_{3}(690 \mathrm{mg}, 5 \mathrm{mmol})$ were added into acetonitrile $(20 \mathrm{~mL})$ with stirring. The mixture was heated at $70{ }^{\circ} \mathrm{C}$ under $\mathrm{N}_{2}$ atmosphere for $24 \mathrm{~h}$. After cooling down to room temperature, the reaction mixture was filtered and the residue was washed with $\mathrm{CH}_{2} \mathrm{Cl}_{2}$. Then, the filtrate was concentrated under reduced pressure. The residue was dissolved by $\mathrm{CH}_{2} \mathrm{Cl}_{2}(50 \mathrm{~mL})$ and washed twice with saturated $\mathrm{NaCl}$ solution. The organic phase was concentrated. The crude product was purified by column chromatography over silica gel (eluent: petroleum ether/ethyl acetate $=20: 1$ ), and compound $\mathbf{4}$ was obtained as white powder in $80 \%$ yield. ${ }^{1} \mathrm{H}$ NMR $\left(400 \mathrm{MHz}, \mathrm{CDCl}_{3}, \mathrm{ppm}\right): \delta 7.75$ $(\mathrm{d}, J=8.5 \mathrm{~Hz}, 2 \mathrm{H}), 6.90(\mathrm{~d}, J=8.5 \mathrm{~Hz}, 2 \mathrm{H}), 4.32(\mathrm{t}, J=6.4 \mathrm{~Hz}, 2 \mathrm{H}), 3.64(\mathrm{t}, J=$ $6.3 \mathrm{~Hz}, 2 \mathrm{H}), 1.33(\mathrm{~s}, 12 \mathrm{H}) .{ }^{13} \mathrm{C} \mathrm{NMR}\left(100 \mathrm{MHz} \mathrm{CDCl}_{3}, \mathrm{ppm}\right): \delta 160.6,136.6$, 114.0, 83.6, 67.6, 28.9, 24.9. HRMS $[\mathrm{M}+\mathrm{H}]+$ calcd. for $\mathrm{C}_{14} \mathrm{H}_{21} \mathrm{BBrO}_{3}+327.0767$; found: 327.0754; Anal. Cald. for $\mathrm{C}_{14} \mathrm{H}_{20} \mathrm{BBrO}_{3}$ : C, 51.42; H, 6.16; Found: C, 51.38; H, 6.18.

Synthesis of compound 2. Compound 4 (327 mg, $1 \mathrm{mmol})$, compound 3 (210 mg, $0.4 \mathrm{mmol}), \mathrm{Pd}\left(\mathrm{PPh}_{3}\right)_{4}(70 \mathrm{mg}, 0.06 \mathrm{mmol})$, and $\mathrm{Na}_{2} \mathrm{CO}_{3}(680 \mathrm{mg}, 6.4 \mathrm{mmol})$ were added to a mixed solution of water $(4 \mathrm{~mL})$ and dimethoxyethane $(30 \mathrm{~mL})$. The mixture was refluxed under $\mathrm{N}_{2}$ at $90{ }^{\circ} \mathrm{C}$ in dark for $24 \mathrm{~h}$. After cooling down to room temperature, the solvent was removed under vacuum. The residue was extracted by dichloromethane, and purified on a silica gel column using petroleum ether/ethyl acetate $(20: 1)$ as the eluent. ${ }^{1} \mathrm{H}$ NMR $\left(400 \mathrm{MHz}, \mathrm{CDCl}_{3}, \mathrm{ppm}\right): \delta 7.47$ $(\mathrm{d}, J=8.6 \mathrm{~Hz}, 4 \mathrm{H}), 7.17(\mathrm{~s}, 2 \mathrm{H}), 6.93(\mathrm{~d}, J=8.6 \mathrm{~Hz}, 4 \mathrm{H}), 4.32(\mathrm{t}, J=6.2 \mathrm{~Hz}, 4 \mathrm{H})$, $3.66(\mathrm{t}, J=6.2 \mathrm{~Hz}, 4 \mathrm{H}), 1.94(\mathrm{~s}, 6 \mathrm{H}) .{ }^{13} \mathrm{C} \mathrm{NMR}\left(100 \mathrm{MHz}, \mathrm{CDCl}_{3}, \mathrm{ppm}\right): \delta 158.0$, $141.9,140.5,127.0,126.9,125.8,121.5,115.2,68.0,28.9,14.5$. HRMS $[\mathrm{M}+\mathrm{H}]^{+}$ calcd. for $\mathrm{C}_{31} \mathrm{H}_{25} \mathrm{Br}_{2} \mathrm{~F}_{6} \mathrm{O}_{2} \mathrm{~S}_{2}+766.9546$; found: 766.9537; Anal. Cald. for $\mathrm{C}_{31} \mathrm{H}_{24} \mathrm{Br}_{2} \mathrm{~F}_{6} \mathrm{O}_{2} \mathrm{~S}_{2}$ : C, 48.58; H, 3.16; Found: C, 48.51; H, 3.19.

Synthesis of compound OF-1. Compound 2 ( $383 \mathrm{mg}, 0.5 \mathrm{mmol})$ was dissolved in acetonitrile $(10 \mathrm{~mL})$, and then 1 -methylimidazole $(410 \mathrm{mg}, 5 \mathrm{mmol})$ was added. The reaction mixture was stirred at $80{ }^{\circ} \mathrm{C}$ for $12 \mathrm{~h}$. After cooling down to room temperature, the obtained precipitate was collected by centrifugation and washed with diethyl ether for three times to afford the desired product OF-1 in $90 \%$ yield. ${ }^{1} \mathrm{H}$ NMR (400 MHz, DMSO- $\left.d_{6}, \mathrm{ppm}\right): \delta 9.20(\mathrm{~s}, 2 \mathrm{H}), 7.83$ (s, 2H), 7.73 (s, 2H), 7.58 $(\mathrm{d}, J=8.4 \mathrm{~Hz}, 4 \mathrm{H}), 7.39(\mathrm{~s}, 2 \mathrm{H}), 7.02(\mathrm{~d}, J=8.6 \mathrm{~Hz}, 4 \mathrm{H}), 4.61(\mathrm{t}, J=4.6 \mathrm{~Hz}, 4 \mathrm{H})$, $4.39(\mathrm{t}, J=4.7 \mathrm{~Hz}, 4 \mathrm{H}), 3.88(\mathrm{~s}, 6 \mathrm{H}), 1.93(\mathrm{~s}, 6 \mathrm{H}) .{ }^{13} \mathrm{C}$ NMR $\left(100 \mathrm{MHz}, \mathrm{DMSO}-d_{6}\right.$, ppm): $\delta 158.1,142.0,140.7,137.5,127.2,126.4,125.4,124.0,123.3,121.8,115.8$, 66.4, 48.8, 36.3, 14.5. HRMS $[\mathrm{M}-2 \mathrm{Br}]^{2+}$ calcd. for $\mathrm{C}_{39} \mathrm{H}_{36} \mathrm{~F}_{6} \mathrm{~N}_{4} \mathrm{O}_{2} \mathrm{~S}_{2}{ }^{2+} 385.1086$; found: 385.1081. Anal. Cald. for $\mathrm{C}_{39} \mathrm{H}_{36} \mathrm{Br}_{2} \mathrm{~F}_{6} \mathrm{~N}_{4} \mathrm{O}_{2} \mathrm{~S}_{2}$ : C, 50.33; H, 3.90; N, 6.02; Found: C, 50.39; H, 3.98; N, 5.94 .

Preparation of the QR code. In a standard procedure, commercial PET film was first printed with blue background, and the QR-pattern was then directly printed on the blue PET film. The QR code was scanned by a commercially available smartphone APP.

\section{Data availability}

The authors declare that the data supporting the findings of this study are available within the article and its Supplementary Information. Extra data are available from the corresponding authors upon reasonable request. 
Received: 7 September 2020; Accepted: 8 February 2021;

Published online: 01 March 2021

\section{References}

1. Shikha, S., Salafi, T., Cheng, J. \& Zhang, Y. Versatile design and synthesis of nano-barcodes. Chem. Soc. Rev. 46, 7054-7093 (2017).

2. Tsang, M., Bai, G. \& Hao, J. Stimuli responsive upconversion luminescence nanomaterials and films for various applications. Chem. Soc. Rev. 44, 1585-1607 (2015).

3. Kumar, P., Singh, S. \& Gupta, B. K. Future prospects of luminescent nanomaterial based security inks: from synthesis to anti-counterfeiting applications. Nanoscale 8, 14297-14340 (2016).

4. Ren, W., Lin, G., Clarke, C., Zhou, J. \& Jin, D. Optical nanomaterials and enabling technologies for high-security-level anticounterfeiting. Adv. Mater. 32, 1901430 (2019).

5. An, Z. et al. Stabilizing triplet excited states for ultralong organic phosphorescence. Nat. Mater. 14, 685-690 (2015).

6. Jiang, K. et al. Triple-mode emission of carbon dots: applications for advanced anti-counterfeiting. Angew. Chem. Int. Ed. 55, 7231-7235 (2016).

7. Hou, X. et al. Tunable solid-state fluorescent materials for supramolecular encryption. Nat. Commun. 6, 6884 (2015).

8. Liu, X. et al. Binary temporal upconversion codes of $\mathrm{Mn}^{2+}$-activated nanoparticles for multilevel anti-counterfeiting. Nat. Commun. 8, 899 (2017).

9. Lu, Y. et al. Tunable lifetime multiplexing using luminescent nanocrystals. Nat. Photon. 8, 32-36 (2014).

10. Xu, S., Chen, R., Zheng, C. \& Huang, W. Excited state modulation for organic afterglow: materials and applications. Adv. Mater. 28, 9920-9940 (2016).

11. Su, Y. et al. Ultralong room temperature phosphorescence from amorphous organic materials toward confidential information encryption and decryption. Sci. Adv. 4, eaas9732 (2018)

12. Ma, Y. et al. On-demand regulation of photochromic behavior through various counterions for high-level security printing. Sci. $A d v$. 6, eaaz2386 (2020).

13. Song, Z. et al. Invisible security ink based on water-soluble graphitic carbon nitride quantum dots. Angew. Chem. Int. Ed. 55, 2773-2777 (2016).

14. Pan, M. et al. Epitaxial growth of hetero-Ln-MOF hierarchical single crystals for domain- and orientation-controlled multicolor luminescence 3D coding capability. Angew. Chem. Int. Ed. 56, 14582-14586 (2017).

15. $\mathrm{Li}, \mathrm{Z}$. et al. Loading photochromic molecules into a luminescent metal-organic framework for information anticounterfeiting. Angew. Chem. Int. Ed. 58, 18025-18031 (2019).

16. Zhang, C. et al. Conversion of invisible metal-organic frameworks to luminescent perovskite nanocrystals for confidential information encryption and decryption. Nat. Commun. 8, 1138 (2017).

17. Xu, L. et al. Double-protected all-inorganic perovskite nanocrystals by crystalline matrix and silica for triple-modal anti-counterfeiting codes. ACS Appl. Mater. Interfaces 9, 26556-26564 (2017).

18. Li, X. et al. A stimuli-responsive smart lanthanide nanocomposite for multidimensional optical recording and encryption. Angew. Chem. Int. Ed. 56, 2689-2693 (2017).

19. Cui, Y., Yue, Y., Qian, G. \& Chen, B. Luminescent functional metal-organic frameworks. Chem. Rev. 112, 1126-1162 (2012).

20. Eliseeva, S. V. \& Bunzli, J. C. G. Lanthanide luminescence for functional materials and bio-sciences. Chem. Soc. Rev. 39, 189-227 (2010).

21. Rocha, J., Carlos, L. D., Paz, F. A. A. \& Ananias, D. Luminescent multifunctional lanthanides-based metal-organic frameworks. Chem. Soc. Rev. 40, 926-940 (2011).

22. Binnemans, K. Lanthanide-based luminescent hybrid materials. Chem. Rev. 109, 4283-4374 (2009).

23. Liu, J. et al. Simultaneously excited downshifting/upconversion luminescence from lanthanide-doped core/shell fluoride nanoparticles for multimode anticounterfeiting. Adv. Funct. Mater. 28, 1707365 (2018).

24. Li, F., Wang, X., Xia, Z., Pan, C. \& Liu, Q. Photoluminescence tuning in stretchable PDMS film grafted doped core/multishell quantum dots for anticounterfeiting. Adv. Funct. Mater. 27, 1700051 (2017).

25. Tu, D., Zheng, W., Huang, P. \& Chen, X. Europium-activated luminescent nanoprobes: From fundamentals to bioapplications. Coord. Chem. Rev. $\mathbf{3 7 8}$, 104-120 (2017).

26. Ji, X. et al. Encoding, reading, and transforming information using multifluorescent supramolecular polymeric hydrogels. Adv. Mater. 30, 1705480 (2018).

27. Chen, X., Jin, Q., Wu, L., Tung, C. \& Tang, X. Synthesis and unique photoluminescence properties of nitrogen-rich quantum dots and their applications. Angew. Chem. Int. Ed. 53, 12542-12547 (2014).
28. Mcconnell, A. J., Wood, C. S., Neelakandan, P. P. \& Nitschke, J. R. Stimuliresponsive metal-ligand assemblies. Chem. Rev. 115, 7729-7793 (2015).

29. Qu, D.-H., Wang, Q., Zhang, Q., Ma, X. \& Tian, H. Photoresponsive hostguest functional systems. Chem. Rev. 115, 7543-7588 (2015).

30. Hai, J. et al. Reversible response of luminescent terbium(III)-nanocellulose hydrogels to anions for latent fingerprint detection and encryption. Angew. Chem. Int. Ed. 57, 6786-6790 (2018).

31. Sun, H. et al. Smart responsive phosphorescent materials for data recording and security protection. Nat. Commun. 5, 3601 (2014).

32. Lerch, M. M., Szymanski, W. \& Feringa, B. L. The (photo)chemistry of stenhouse photoswitches: Guiding principles and system design. Chem. Soc. Rev. 47, 1910-1937 (2018).

33. Danowski, W. et al. Unidirectional rotary motion in a metal-organic framework. Nat. Nanotechnol. 14, 488-494 (2019).

34. Bleger, D. \& Hecht, S. Visible-light-activated molecular switches. Angew. Chem. Int. Ed. 54, 11338-11349 (2015).

35. Irie, M., Fukaminato, T., Matsuda, K. \& Kobatake, S. Photochromism of diarylethene molecules and crystals: memories, switches, and actuators. Chem. Rev. 114, 12174-12277 (2014)

36. Qi, Q. et al. Solid-state photoinduced luminescence switch for advanced anticounterfeiting and super-resolution imaging applications. J. Am. Chem. Soc. 139, 16036-16039 (2017).

37. Wu, H., Chen, Y. \& Liu, Y. Reversibly photoswitchable supramolecular assembly and its application as a photoerasable fluorescent ink. Adv. Mater 29, 1605271 (2017).

38. Yin, Z. et al. Local field modulation induced three-order upconversion enhancement: combining surface plasmon effect and photonic crystal effect. Adv. Mater. 28, 2518-2525 (2016)

39. Liu, Y., Ai, K. \& Lu, L. Designing lanthanide-doped nanocrystals with both up- and down-conversion luminescence for anti-counterfeiting. Nanoscale $\mathbf{3}$, 4804-4810 (2011)

40. Liu, H. et al. Phase angle encoded upconversion luminescent nanocrystals for multiplexing applications. Nanoscale 9, 1676-1686 (2017)

41. Wen, S. et al. Future and challenges for hybrid upconversion nanosystems. Nat. Photon. 13, 828-838 (2019).

42. Liu, K.-K. et al. Advanced encryption based on fluorescence quenching of $\mathrm{ZnO}$ nanoparticles. J. Mater. Chem. C. 5, 7167-7173 (2017).

43. Tian, H. \& Yang, S. Recent progresses on diarylethene based photochromic switches. Chem. Soc. Rev. 33, 85-97 (2004).

44. Uno, K., Bossi, M. L., Irie, M., Belov, V. N. \& Hell, S. W. Reversibly photoswitchable fluorescent diarylethenes resistant against photobleaching in aqueous solutions. J. Am. Chem. Soc. 141, 16471-16478 (2019).

45. Carling, C.-J., Boyer, J.-C. \& Branda, N. R. Remote-control photoswitching using NIR light. J. Am. Chem. Soc. 131, 10838-10839 (2009).

46. Asadirad, A. M., Boutault, S., Erno, Z. \& Branda, N. R. Controlling a polymer adhesive using light and a molecular switch. J. Am. Chem. Soc. 136, 3024-3027 (2014).

47. Ko, C. \& Yam, V. W.-W. Coordination compounds with photochromic ligands: ready tunability and visible light-sensitized photochromism. Acc. Chem. Res. 51, 149-159 (2018).

48. Cheng, H.-B., Zhang, H.-Y. \& Liu, Y. Dual-stimulus luminescent lanthanide molecular switch based on an unsymmetrical diarylperfluorocyclopentene. J. Am. Chem. Soc. 135, 10190-10193 (2013).

49. Cheng, H.-B. et al. Photocontrolled reversible luminescent lanthanide molecular switch based on a diarylethene-europium dyad. Inorg. Chem. 55, 7962-7968 (2016)

50. Hasegawa, Y., Nakagawa, T. \& Kawai, T. Recent progress of luminescent metal complexes with photochromic units. Coord. Chem. Rev. 254, 2643-2651 (2010).

51. Xu, L. et al. Supramolecular self-assembly enhanced europium(III) luminescence under visible light. Soft Matter 10, 4686-4693 (2014).

52. Wang, J. et al. Response of metal-coordination-based polyelectrolyte complex micelles to added ligands and metals. Soft Matter 16, 2953-2960 (2020).

53. Li, B., Ding, Z.-J., Li, Z. \& Li, H. Simultaneous enhancement of mechanical strength and luminescence performance in double-network supramolecular hydrogels. J. Mater. Chem. C. 6, 6869-6874 (2018).

54. Li, Z., Wang, G., Wang, Y. \& Li, H. Reversible phase transition of robust luminescent hybrid hydrogels. Angew. Chem. Int. Ed. 57, 2194-2198 (2018).

55. Wang, J. et al. A supramolecular crosslinker to give salt-resistant polyion complex micelles and improved MRI contrast agents. Angew. Chem. Int. Ed. 57, 12680-12684 (2018)

56. Wang, J. et al. Processable and luminescent supramolecular hydrogels from complex coacervation of polycations with lanthanide coordination polyanions. Macromolecules 52, 8643-8650 (2019).

57. Vermonden, T. et al. Water-soluble reversible coordination polymers: chains and rings. Macromolecules 36, 7035-7044 (2003).

58. Vermonden, T. et al. Linear rheology of water-soluble reversible neodymium (III) coordination polymers. J. Am. Chem. Soc. 126, 15802-15808 (2004). 
59. Wang, J. et al. Controlled mixing of lanthanide(III) ions in coacervate core micelles. Chem. Commun. 49, 3736-3738 (2013).

60. Zhou, W. et al. Functional polyion complex vesicles enabled by supramolecular reversible coordination polyelectrolytes. Angew. Chem. Int. Ed. 58, 8494-8498 (2019).

61. Yan, Y. et al. Hierarchical self-assembly in solutions containing metal ions, ligand, and diblock copolymer. Angew. Chem. Int. Ed. 46, 1807-1809 (2007).

62. Liu, G., Zhang, Y.-M., Xu, X., Zhang, L. \& Liu, Y. Optically switchable luminescent hydrogel by synergistically intercalating photochromic molecular rotor into inorganic clay. Adv. Opt. Mater. 5, 1700149 (2017).

63. Liu, G., Zhang, Y.-M., Wang, C. \& Liu, Y. Dual visible light-triggered photoswitch of a diarylethene supramolecular assembly with cucurbit[8]uril. Chem. Eur. J. 23, 14425-14429 (2017).

64. Li, Z. et al. Synthesis, photophysical properties and NIR photochromism of photoresponsive difluoroboron $\beta$-diketonate complex based on dithienylethene unit. Dyes Pigm. 160, 597-603 (2019).

65. Li, Z. et al. Photoresponsive luminescent polymeric hydrogels for reversible information encryption and decryption. Adv. Sci. 6, 1901529 (2019).

66. Feng, T. et al. A robust mixed-lanthanide polyMOF membrane for ratiometric temperature sensing. Angew. Chem. Int. Ed. 59, 21752-21757 (2020).

67. Li, Z.-Q., Zhang, Y.-M., Guo, D.-S., Chen, H.-Z. \& Liu, Y. Supramolecular assembly with multiple preorganised $\pi$-electronic cages. Chem. Eur. J. 19, 96-100 (2013)

68. Irie, M., Lifka, T., Kobatake, S. \& Kato, N. Photochromism of 1,2-bis(2methyl-5-phenyl-3-thienyl)perfluorocyclopentene in a single-crystalline phase. J. Am. Chem. Soc. 122, 4871-4876 (2000).

69. Nakahama, T., Kitagawa, D. \& Kobatake, S. Tuning of optical properties and thermal cycloreversion reactivity of photochromic diarylbenzene by introducing electron-donating substituents. J. Phys. Chem. C. 123, 31212-31218 (2019).

\section{Acknowledgements}

This work was financially supported by the National Natural Science Foundation of China $(21871075,21502039$, and 21771050), the Natural Science Foundation of Hebei Province (B2018202134, B2016202149, B2016202147, and B2017202048), the Tianjin Natural Science Foundation (19JCQNJC04900), the Program for Top 100 Innovative Talents in Colleges and Universities of Hebei Province (SLRC2019023), the Singapore Academic Research Fund (RT12/19), and the Singapore Agency for Science, Technology, and Research (A*STAR) AME IRG grant (A1883c0005).

\section{Author contributions}

Z.L. designed the experiments and drafted the manuscript. Z.L., X.L., G.W., B.L., and H.C. performed the experiments and analyzed the data. H.L. and Y.Z. supervised the work and edited the manuscript.

\section{Competing interests}

The authors declare no competing interests.

\section{Additional information}

Supplementary information The online version contains supplementary material available at https://doi.org/10.1038/s41467-021-21677-4.

Correspondence and requests for materials should be addressed to Z.L., H.L. or Y.Z.

Peer review information Nature Communications thanks Alexander Kuehne and the other, anonymous, reviewer(s) for their contribution to the peer review of this work. Peer reviewer reports are available.

Reprints and permission information is available at http://www.nature.com/reprints

Publisher's note Springer Nature remains neutral with regard to jurisdictional claims in published maps and institutional affiliations.

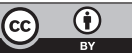

Open Access This article is licensed under a Creative Commons Attribution 4.0 International License, which permits use, sharing, adaptation, distribution and reproduction in any medium or format, as long as you give appropriate credit to the original author(s) and the source, provide a link to the Creative Commons license, and indicate if changes were made. The images or other third party material in this article are included in the article's Creative Commons license, unless indicated otherwise in a credit line to the material. If material is not included in the article's Creative Commons license and your intended use is not permitted by statutory regulation or exceeds the permitted use, you will need to obtain permission directly from the copyright holder. To view a copy of this license, visit http://creativecommons.org/ licenses/by/4.0/.

(C) The Author(s) 2021 